\title{
Mesenchymal stem cells suppress CaN/NFAT expression in the pulmonary arteries of rats with pulmonary hypertension
}

\author{
JUNFENG LIU ${ }^{1,2}$, ZHIBO HAN ${ }^{3}$, ZHONGCHAO HAN ${ }^{3}$ and ZHIXU HE ${ }^{1}$ \\ ${ }^{1}$ Laboratory of Tissue Engineering and Stem Cells, Guiyang Medical College, Guiyang, Guizhou 550004; \\ ${ }^{2}$ Department of Pediatrics, The General Hospital of Huabei Oil Field Company, Renqiu, Hebei 062552; \\ ${ }^{3}$ National Engineering Research Center of Cell Products, AmCellGene Co. Ltd., Tianjin 300457, P.R. China
}

Received November 15, 2014; Accepted August 13, 2015

DOI: $10.3892 /$ etm.2015.2722

\begin{abstract}
Inflammation and hyperproliferation of pulmonary artery smooth muscle cells (PASMCs) is considered the primary pathological feature of pulmonary hypertension $(\mathrm{PH})$. The present study determined that mesenchymal stem cells (MSCs) suppress the expression of calcineurin (CaN) and nuclear factor of activated T-cells (NFAT) in the pulmonary arteries of rats, and this may exert a therapeutic effect on $\mathrm{PH}$. The potential therapeutic effects of MSCs on PH were assessed via the transplantation of human umbilical cord-derived MSCs, which were cultured in serum-free medium, into a monocrotaline (MCT)-induced PH rat model. Subsequently, the expression levels of tumor necrosis factor (TNF)- $\alpha$ in lung tissue and plasma, and of $\mathrm{CaN}$ and NFATc2 in pulmonary arteries were assessed. In the rat model of MCT-induced $\mathrm{PH}$, investigated in the present study, TNF- $\alpha$ expression levels were detected in the lung tissue, and the levels of TNF- $\alpha$ in the plasma were increased. Furthermore, in addition to hemodynamic changes and the evident medial hypertrophy of the pulmonary muscular arterioles, $\mathrm{CaN}$ and NFATc2 expression levels were significantly upregulated in the pulmonary arteries. In the present study, the transplantation of MSCs, cultured in serum-free medium, decreased the levels of TNF- $\alpha$ in the lung tissue and plasma of rats, and downregulated $\mathrm{CaN}$ and NFATc 2 expression in the pulmonary arteries. Furthermore, hemodynamic abnormalities and medial hypertrophy of the pulmonary muscular arterioles were notably improved. Therefore, the results of the present study may suggest that the administration of MSCs in PH may suppress the production of
\end{abstract}

Correspondence to: Professor Zhixu He, Laboratory of Tissue Engineering and Stem Cells, Guiyang Medical College, 4 Beijing Road, Guiyang, Guizhou 550004, P.R. China

E-mail:hzx@gmc.edu.cn

Professor Zhongchao Han, National Engineering Research Center of Cell Products, AmCellGene Co. Ltd., 4th Street TEDA, Tianjin 300457, P.R. China

E-mail: hanzhongchao@hotmail.com

Key words: pulmonary hypertension, mesenchymal stem cell, calcineurin, nuclear factor of activated T-cells, model, transplantation
TNF- $\alpha$, and downregulate the expression of CaN and NFATc2 in pulmonary arteries, which may provide an effective treatment for PH by suppressing the pathological proliferation of PASMCs.

\section{Introduction}

Pulmonary hypertension (PH) is defined as an increase in pulmonary artery resistance and vascular remodeling, which can result in right ventricular overload and heart failure. Although the clinical usage of targeted drugs, such as Sildenafil and bosentan, may partly ameliorate the symptoms of $\mathrm{PH}$, the long-term outcomes remain poor. Therefore, a more effective medicine or therapeutic protocol is required. Previous studies have indicated that inflammation participates in the pathological mechanism of $\mathrm{PH}$, and thus may be a promising therapeutic target for the treatment of PH $(1,2)$. Furthermore, tertiary lymphoid follicles and inflammatory infiltration has been demonstrated near the remodeled pulmonary arteries of animal models and patients with $\mathrm{PH}$ (2-5). These inflammatory cells produce chemokines and cytokines, including tumor necrosis factor (TNF)- $\alpha$, and interleukin (IL)-6, and elevated levels of inflammatory cytokines have been reported to act as predictive factors for the survival of patients with idiopathic and familial PH (6). Among the elevated inflammatory cytokines, TNF- $\alpha$ has a crucial role in the hyperproliferation of pulmonary artery smooth muscle cells (PASMCs) and vascular remodeling. Furthermore, previous studies have demonstrated that the calcineurin $(\mathrm{CaN}) /$ nuclear factor of activated T-cells (NFAT) signaling pathway is one of the critical pathways involved in the proliferation of SMCs in $\mathrm{PH}(7,8)$, and thus may be a therapeutic target for the treatment of PH (8). CaN/NFAT in the cytoplasm of SMCs may be activated by increased calcium influx, which subsequently initiates the hyperproliferation of SMCs (9), resulting in vascular remodeling. As previously demonstrated, TNF- $\alpha$ may increase calcium influx, which may result in further activation of $\mathrm{CaN} / \mathrm{NFAT}$, and thus promote the hyperproliferation of SMCs (10). Therefore, the authors of the present study hypothesized that decreasing the production of TNF- $\alpha$ may effectively block the activation of CaN/NFAT and SMCs hyperproliferation, and may be considered a potential treatment for patients with $\mathrm{PH}$. 
Mesenchymal stem cells (MSCs) are a group of primitive cells, which have self-renewal ability and the potential to differentiate along various lineages. The clinical application of MSCs is promising due to the low potential for immunogenicity and graft rejection reactions. Furthermore, a serum-free culture system for the expansion of MSCs may further decrease the potential for zoonotic infections and immunological reactions (11). The authors of the present study have previously demonstrated that MSCs may produce high levels of prostaglandin E2 (PGE2) and immunoregulatory cytokines, and decrease the production of Th1-associated inflammatory cytokines such as TNF- $\alpha$ and interferon- $\gamma$, when co-cultured with activated $\mathrm{CD} 4^{+} \mathrm{T}$ cells (12). The unique immunosuppressive effects produced by MSCs highlight them as a potential treatment for immune-associated diseases $(13,14)$. Previous studies have demonstrated that administration of MSCs may relieve PH in an experimental animal model $(15,16)$; however, the effects of MSCs on the immune disorder and hyperproliferation of PASMCs have yet to be elucidated.

In the present study, a rat model of $\mathrm{PH}$ was induced by a single subcutaneous injection of monocrotaline (MCT), prior to subsequent transplantation of serum-free cultured MSCs derived from a human umbilical cord. The therapeutic effects, and the expression levels of TNF- $\alpha$ and CaN/NFAT in the pulmonary arteries were evaluated, in order to determine the effects of MSCs on the immune disorder and hyperproliferation of PASMCs associated with PH.

\section{Materials and methods}

Human umbilical cord-derived MSCs. The present study was approved by the Institutional Review Board of Guiyang Medical College (Guiyang, China), and written informed consent was obtained from all donors prior to the initiation of this study. Human umbilical cord-derived MSCs were isolated according to our previous study (17) and were cultured in DMEM/F-12 medium (Gibco Life Technologies, Carlsbad, CA, USA) supplemented with $100 \mathrm{U} / \mathrm{ml}$ penicillin-streptomycin and $10 \%$ fetal bovine serum (FBS; HyClone, GE Healthcare Life Sciences, Logan, UT, USA). On the third passage, MSC culture medium was exchanged for serum-free Chemically Defined Mesenchymal Stem Cell Medium (Lonza Inc., Allendale, NJ, USA). MSCs at passage 8-10 were identified by analyzing the ability of osteogenic and adipogenic differentiation, as described previously (11). In addition, the expression of cell-surface markers, including CD19, CD29, CD31, CD34, CD44, CD73, CD90, CD105, human leukocyte antigen (HLA)-DR and HLA-ABC was detected using flow cytometry (BD Biosciences, Franklin Lakes, NJ, USA) (17).

Experimental animals. A total of 24 female Sprague-Dawley rats (body weight, $200 \mathrm{~g}$ ) were purchased and housed in specific pathogen-free units at the Laboratory Animals Center at Tianjin Blood Diseases Hospital (Tianjin, China). The rats were maintained at $25^{\circ} \mathrm{C}$ in $70 \%$ relative humidity, with a 12-h light/dark cycle. All animal studies were approved by the Institutional Animal Care and Use Committee of Guiyang Medical College. The 24 rats were randomly divided into three equal groups: The model group [(MCT and phosphate-buffered saline (PBS)], the treatment group (MCT and MSCs), and the control group (PBS). PH was induced in the rats by a single subcutaneous injection of $60 \mathrm{mg} / \mathrm{kg}$ MCT (Sigma-Aldrich, St. Louis, MO, USA) (18), with PBS administered as a control. A total of 5 days after MCT injection, PBS or MSCs were administered via the caudal vein. Briefly, at passage 8-10, the MSCs were detached for transplantation using $0.25 \%$ trypsin (Gibco Life Technologies) and $0.53 \mathrm{mM}$ EDTA (Sigma-Aldrich). Following subsequent washing with PBS twice, $10^{6}$ cells were resuspended in $1 \mathrm{ml}$ PBS, and transplanted into the rats via the caudal vein, 5 days after injection of MCT. Furthermore, in order to observe the distribution of MSCs in the lungs, two additional rats received transplantation of CM-Dil-labeled (Invitrogen Life Technologies, Carlsbad, CA, USA) MSCs under the same protocol.

Examination of hemodynamics. At day 21, the rats were anesthetized by intraperitoneal injection of $50 \mathrm{mg} / \mathrm{kg}$ pentobarbital (Sigma-Aldrich). Polygraph examination (Nihon Kohden Corporation, Tokyo, Japan) of the mean aortic pressure (MAoP) was performed by inserting a polyethylene catheter into the ascending aorta via the right carotid artery. The right ventricular systolic pressure (RVSP) was subsequently tested by inserting a polyethylene catheter into the right ventricle via the right external jugular vein. Subsequently, blood samples were collected from the external jugular vein. Plasma was segregated by centrifugation at $2,000 \mathrm{xg}$ for $10 \mathrm{~min}$ at $4{ }^{\circ} \mathrm{C}$, and stored at $-80^{\circ} \mathrm{C}$. The rats were subsequently sacrificed by decapitation, prior to harvesting of the lung tissue and pulmonary arteries. The pulmonary arteries were immersed in RNAsafer Stabilizer Reagent (Tianjin Baorui Biological Technology Co., Ltd., Tianjin, China), and the remaining pulmonary lobes were fixed in 10\% paraformaldehyde (Sigma-Aldrich) at room temperature.

Histological examination. Following fixation in $10 \%$ paraformaldehyde for $24 \mathrm{~h}$, the lung tissue was embedded in paraffin, and serial $5 \mu \mathrm{m}$ sections were stained with hematoxylin and eosin (Yuanmu Biotechnology Co., Ltd., Shanghai, China), prior to observation under a fluorescence microscope (Olympus Corporation, Tokyo, Japan). The medial wall thickness of the pulmonary arteriole is expressed as: Wall thickness $(\mathrm{WT} ; \%)=[($ medial thickness x 2$) /$ external diameter] x 100 (19).

TNF- $\alpha$ expression in the lung tissue. Paraffin sections fixed on gelatin-coated slides were deparaffinized and rehydrated prior to sequential incubation with $0.3 \%$ Triton X-100 (Sigma-Aldrich), 3\% hydrogen peroxide (Santa Cruz Biotechnology Inc., Dallas, TX, USA) and $2 \%$ bovine serum albumin (MP Biomedicals, Santa Ana, CA, USA). The sections were subsequently incubated overnight at $4^{\circ} \mathrm{C}$ with a goat polyclonal primary antibody against TNF- $\alpha$ (1:400; sc-1350; Santa Cruz Biotechnology Inc.). The following day, the lung tissue was incubated with a biotinylated rabbit anti-goat secondary antibody (Wuhan Boster Biotechnology Co., Ltd., Wuhan, China) for $30 \mathrm{~min}$, prior to immunoreactivity detection using a 3-amino-9-ethylcarbazole peroxidase substrate kit (Wuhan Boster Biotechnology Co., Ltd.). 

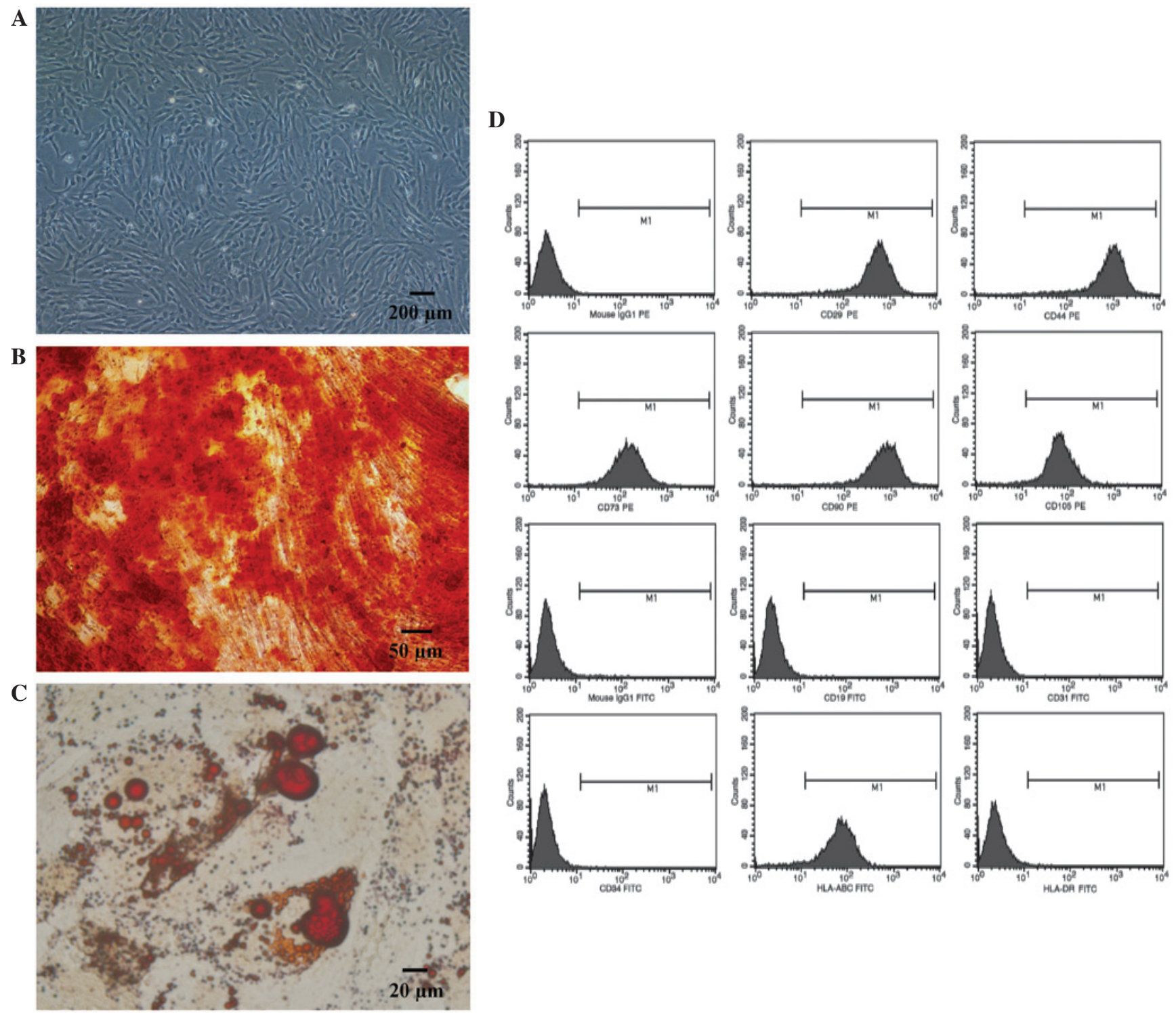

Figure 1. Identification of mesenchymal stem cells (MSCs). (A) Passage 3 MSCs cultured in vitro. (B) Osteogenic differentiation of MSCs was identified using Alizarin red S stain. (C) Adipogenic differentiation of MSCs was identified using Oil Red O stain. (D) Phenotypic analysis of MSCs.

TNF- $\alpha$ plasma levels. The plasma levels of TNF- $\alpha$ were determined using an ELISA kit (PeproTech, Inc., Rocky Hill, NJ, USA), according to the manufacturer's instructions.

CaN and NFATc2 expression in the pulmonary arteries. Total RNA was extracted from the pulmonary arteries using an E.Z.N.A. Total RNA I kit (Omega Biotek, Inc., Norcross, GA, USA), which included tissue lysing and homogenization, according to the manufacturer's instructions. Subsequently, the total RNA was reverse transcribed into cDNA using a Moloney Murine Leukemia Virus Reverse Transcriptase kit (Invitrogen Life Technologies). Reverse transcription-quantitative polymerase chain reaction (RT-qPCR) analyses for $\mathrm{CaN}$ and NFATc2 were performed on an 7300 Real-Time PCR system (Applied Biosystems Life Technologies, Foster City, CA, USA) using Platinum ${ }^{\circledR}$ SYBR $^{\circledR}$ Green qPCR SuperMix-UDG with ROX (Invitrogen Life Technologies). The primer sequences were as follows: GAPDH, forward 5'-CCATTCTTCCAC CTTTGATGCT-3', reverse 5'-TGTTGCTGTAGCCATATT
CATTGT-3'; CaN, forward 5'-CAGAGGGTGCTTCGA TTCTC-3', reverse 5'-CCCCTAAGAAGAGGTAGCGA-3'; and NFATc2, forward 5'-CAGCAGATTTGGGAGATGGAA G-3', and reverse 5'-GACTGGGTGGTAAGTAAAGTGC-3'. PCR cycling conditions were as follow: $95^{\circ} \mathrm{C}$ for $2 \mathrm{~min}$, and $95^{\circ} \mathrm{C}$ for $15 \mathrm{sec}$, and $60^{\circ} \mathrm{C}$ for $30 \mathrm{sec}$, for 40 cycles. The relative expression levels were quantified by the $2^{-\Delta \Delta \mathrm{Ct}}$ method.

Statistical analysis. Data are presented as the mean \pm standard deviation. SPSS software (version 17.0; SPSS, Inc., Chicago, IL, USA) was used to perform statistical analyses. Differences were compared using one-way analysis of variance. $\mathrm{P}<0.05$ was considered to indicate a statistically significant difference.

\section{Results}

Identification of MSCs. Human umbilical cord-derived MSCs proliferated well when cultured in serum-free medium. Furthermore, the MSCs exhibited a typical 
A

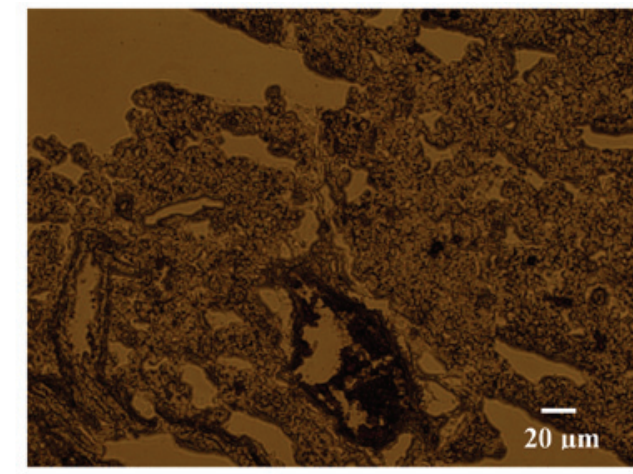

B

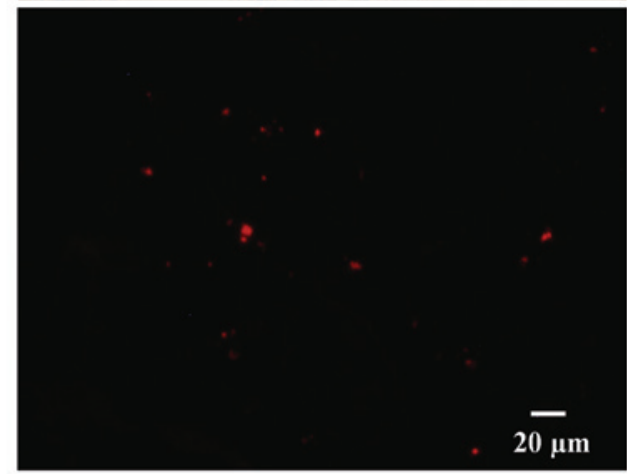

C

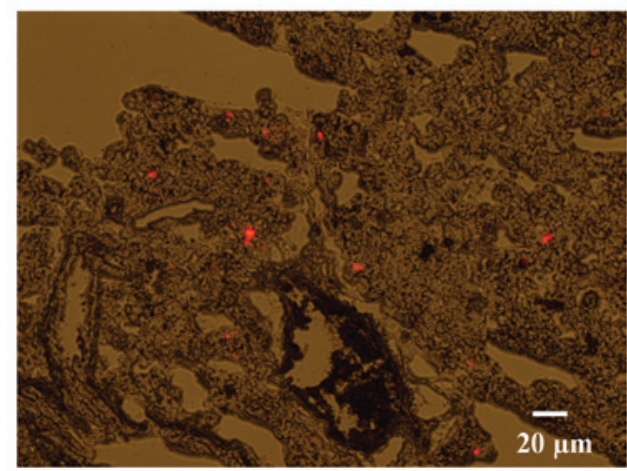

D

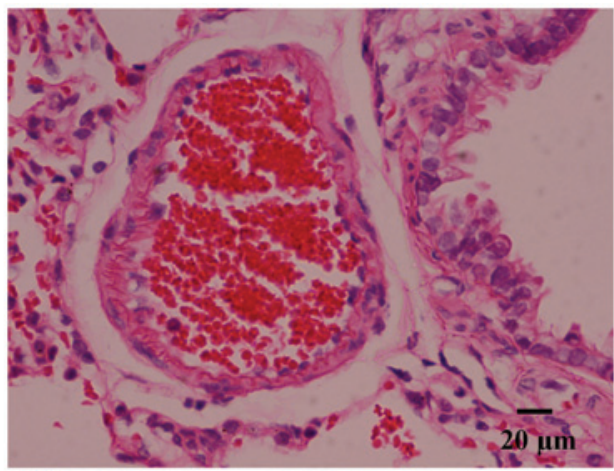

$\mathbf{E}$

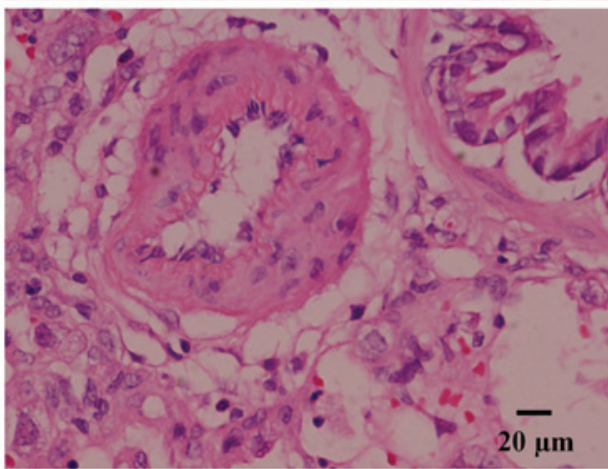

$\mathbf{F}$

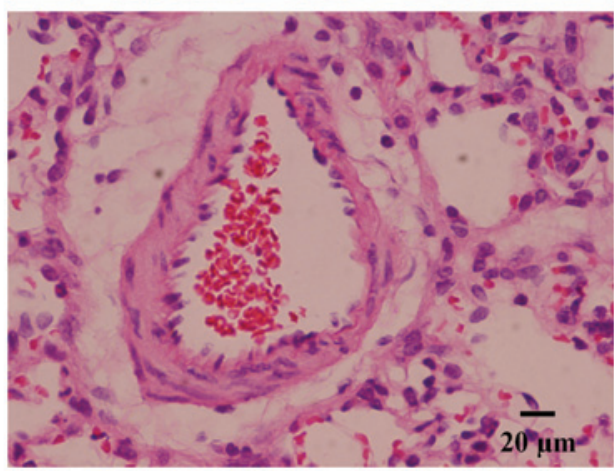

Figure 2. Effects of mesenchymal stem cells (MSCs) on pulmonary hypertension. (A-C) CM-Dil labeled MSCs distributed in the lung 2 days following transplantation; (A) DIC, (B) CM-Dil and (C) merge. (D-F) Optical photomicrographs of lung tissue cells stained with hematoxylin and eosin. (D) Control group; (E) model group; (F) MSCs transplantation group.

fibroblast shape (Fig. 1A), and demonstrated osteogenic (Fig. 1B) and adipogenic (Fig. 1C) differentiation abilities, when cultured in specific conditioned medium. Flow cytometric analysis determined the cells were positive for HLA-ABC, CD29, CD44, CD73, CD90 and CD105, whereas they were negative for: CD19, CD31, CD34 and HLA-DR (Fig. 1D).

Transplantation of MSCs improves $P H$. As described, two rats were transplanted with CM-Dil labeled MSCs; and following a period of 2 days, the rats were sacrificed by decapitation, and the lung tissues were harvested. Serial $5 \mu \mathrm{m}$ cryosections were prepared and observed under a fluorescence microscope; the results of which revealed an abundance of CM-Dil positive cells distributed in the lungs, which may confirm that transplanted MSCs localize to injured lung tissue following transplantation, via a peripheral vessel (Fig. 2A-C). A hemodynamic examination was performed 21 days after subcutaneous injection of MCT, the results of which indicated that the MAoP in the model group was significantly decreased, as compared with that in the control group $(94.64 \pm 14.83$ vs. $118.34 \pm 15.67 \mathrm{mmHg}$; $\mathrm{P}<0.05)$ (Fig. $3 \mathrm{~A})$. The RVSP increased from $27.28 \pm 2.96 \mathrm{mmHg}$ in the control group to $47.13 \pm 3.31 \mathrm{mmHg}$ in the model group $(\mathrm{P}<0.01)$ (Fig. $3 \mathrm{~B})$. Furthermore, compared with that of the model group, the MAoP recovered to $114.25 \pm 13.93 \mathrm{mmHg}(\mathrm{P}<0.05$; Fig. $3 \mathrm{~A})$, whereas the RVSP recovered to $29.86 \pm 2.87 \mathrm{mmHg}(\mathrm{P}<0.01$; Fig. 3B) following MSC treatment. These results suggest that the MSC transplantation treatment improved hemodynamic abnormalities.

In addition to the hemodynamic assessment, a histological examination detected medial hypertrophy of the pulmonary muscular arterioles, in the model group. In the model group, the medial hypertrophy of the pulmonary muscular arterioles (Fig. 2E) was evident compared with the control group (Fig. 2D). the WT increased from $13.48 \pm 1.23$ to $31.78 \pm 2.16 \%(\mathrm{P}<0.01)$; whereas the medial hypertrophy improved following transplantation with the MSCs (Fig. 2F), (WT, 17.53 \pm 1.24 vs. $31.78 \pm 2.16 \%$; $\mathrm{P}<0.01$ ) (Fig. 3C), which was similar to the effects observed of MSCs on hemodynamics. 

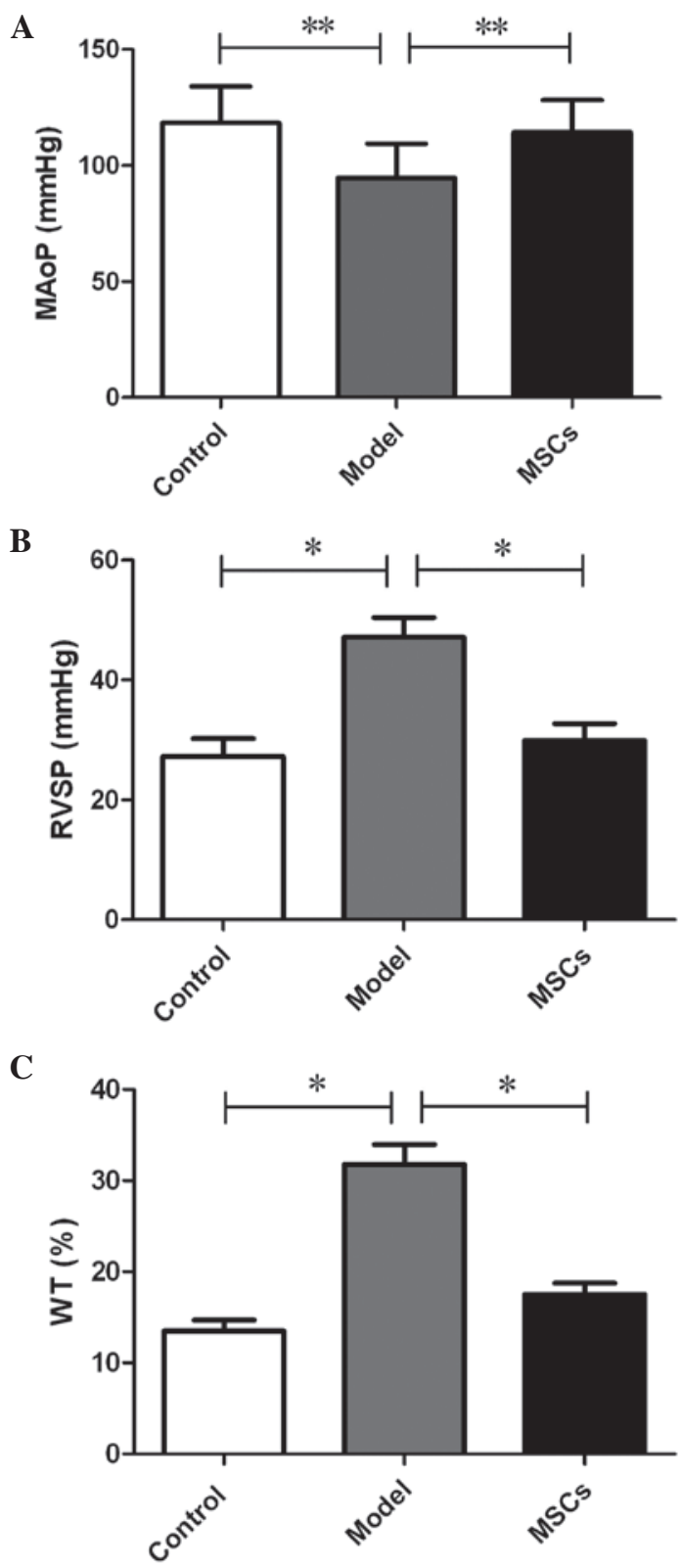

Figure 3. Changes in the (A) mean aortic pressure (MAoP), (B) right ventricular systolic pressure (RVSP) and (C) the medial wall thickness of the pulmonary arteriole (WT) in each group $(n=8)$. Data are presented as the mean \pm standard deviation,.. $\mathrm{P}<0.01,{ }^{* *} \mathrm{P}<0.05$.

MSCs decrease the expression levels of TNF- $\alpha$ in lung tissue and plasma. TNF- $\alpha$ expression was detected in the lung tissue of the PH rat model 21 days after injection with MCT. Immunohistochemistry demonstrated a downregulation of TNF- $\alpha$ expression in the lung tissue of rats transplanted with MSCs, and the average optical density (AOD) decreased from $0.25 \pm 0.06$ in the model group to $0.17 \pm 0.03(\mathrm{P}<0.01)$ (Fig. 4A-D). In addition, plasma TNF- $\alpha$ levels were also measured, and were markedly increased in the model group, as compared with the controls ( $854.49 \pm 80.15$ vs. $135.96 \pm 8.98, \mathrm{P}<0.01$ ) (Fig. 4E). Consistent with the changes in TNF- $\alpha$ expression observed in the lung tissue, TNF- $\alpha$ expression in the plasma of the MSCs treatment group decreased significantly, as compared with the model group $(230.04 \pm 33.22$ vs. $854.49 \pm 80.15, \mathrm{P}<0.01)$, and no differences were found in comparison with the controls $(\mathrm{P}>0.05)$ (Fig. 4E).

MSCs decrease the expression levels of CaN and NFATc2. In order to assess the proliferation of PASMCs, the expression levels of $\mathrm{CaN}$ and NFATc2, which are considered to be critical factors in the proliferation of SMCs, were assessed by RT-qPCR. A total of 21 days after injection with MTC, CaN and NFATc2 expression levels in the pulmonary arteries were significantly upregulated in the model group, as compared with that of the control group (Fig. 4F and G). However, the high expression levels of CaN and NFATc2 were inhibited following transplantation with the MSCs.

\section{Discussion}

Using a rat model of MCT-induced $\mathrm{PH}$, the present study has demonstrated that TNF- $\alpha$ expression was increased in the plasma and lung tissue. Furthermore, in addition to the medial hypertrophy of the pulmonary muscular arterioles and the hemodynamic alterations observed, the expression levels of $\mathrm{CaN}$ and NFATc2 in the pulmonary arteries were significantly upregulated. The transplantation of MSCs cultured in serum-free medium decreased TNF- $\alpha$ expression levels in both the lung tissue and plasma of rats with MCT-induced $\mathrm{PH}$; and the expression levels of $\mathrm{CaN}$ and NFATc2 in the pulmonary arteries were also downregulated. The results of the present study indicated that transplantation with MSCs may improve the medial hypertrophy of pulmonary muscular arterioles and hemodynamic abnormalities associated with $\mathrm{PH}$. Furthermore, the administration of MSCs may provide a potential treatment for patients with PH by suppressing the production of TNF- $\alpha$ and downregulating $\mathrm{CaN}$ and NFATc2 expression in the pulmonary arteries, in order to prevent PASMCs hyperproliferation.

The persistent contraction and remodeling of pulmonary arterioles is the main pathological and physiopathological characteristic of $\mathrm{PH}$, and high blood pressure in the pulmonary circulation may result in of right ventricular overload. With the progression of the disease, right ventricular hypertrophy and dilatation ensue. Despite the development of targeted drugs that may improve the symptoms of $\mathrm{PH}$ and decrease the occurrence of heart attack to a certain extent, heart failure and mortality remain the inevitable fate of numerous patients with $\mathrm{PH}$, as drug efficacy remains limited in the long-term $(20,21)$. Therefore, it is crucial that more effective therapeutic protocols are explored, particularly those that directly target the pathomechanism of PH. With the advancement of studies exploring the mechanisms of $\mathrm{PH}$, various studies have demonstrated that inflammation and hyperproliferation of PASMCs may be a promising target for the treatment of $\mathrm{PH}$, as they are considered to be the major pathological features of PH $(1,8)$. Concordantly, the present study demonstrated that the expression levels of TNF- $\alpha$ in the lung tissue and plasma were increased in the rat model of MCT-induced PH. Furthermore, as pivotal regulatory factors of the proliferation of SMCs, the expression levels of $\mathrm{CaN}$ and NFATc2 in pulmonary arteries were significantly upregulated. Medial hypertrophy of the pulmonary muscular arterioles accompanies these changes, as pulmonary circulation pressure is increased. As a previous 
A

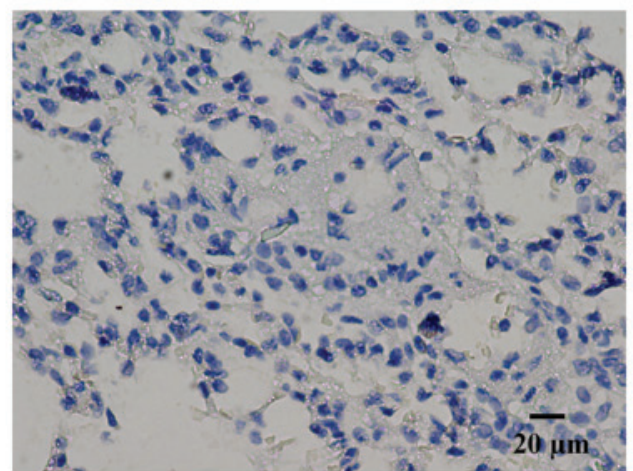

B

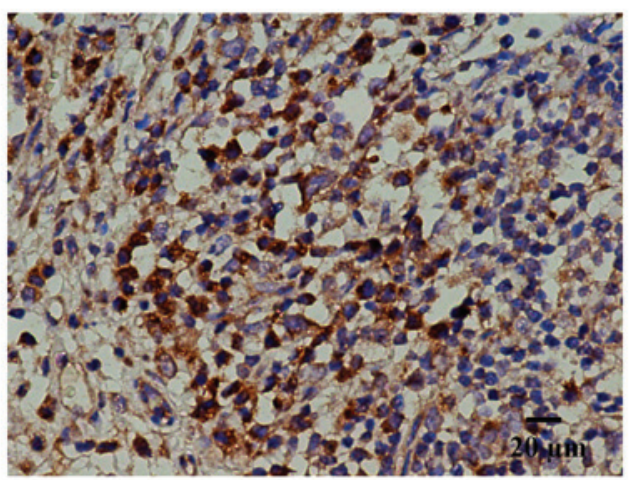

C

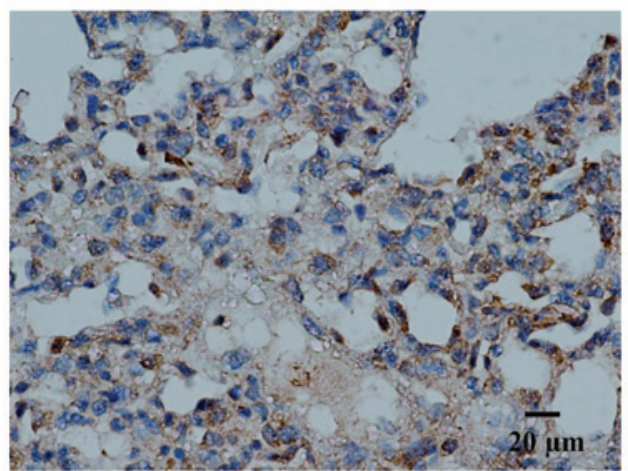

D
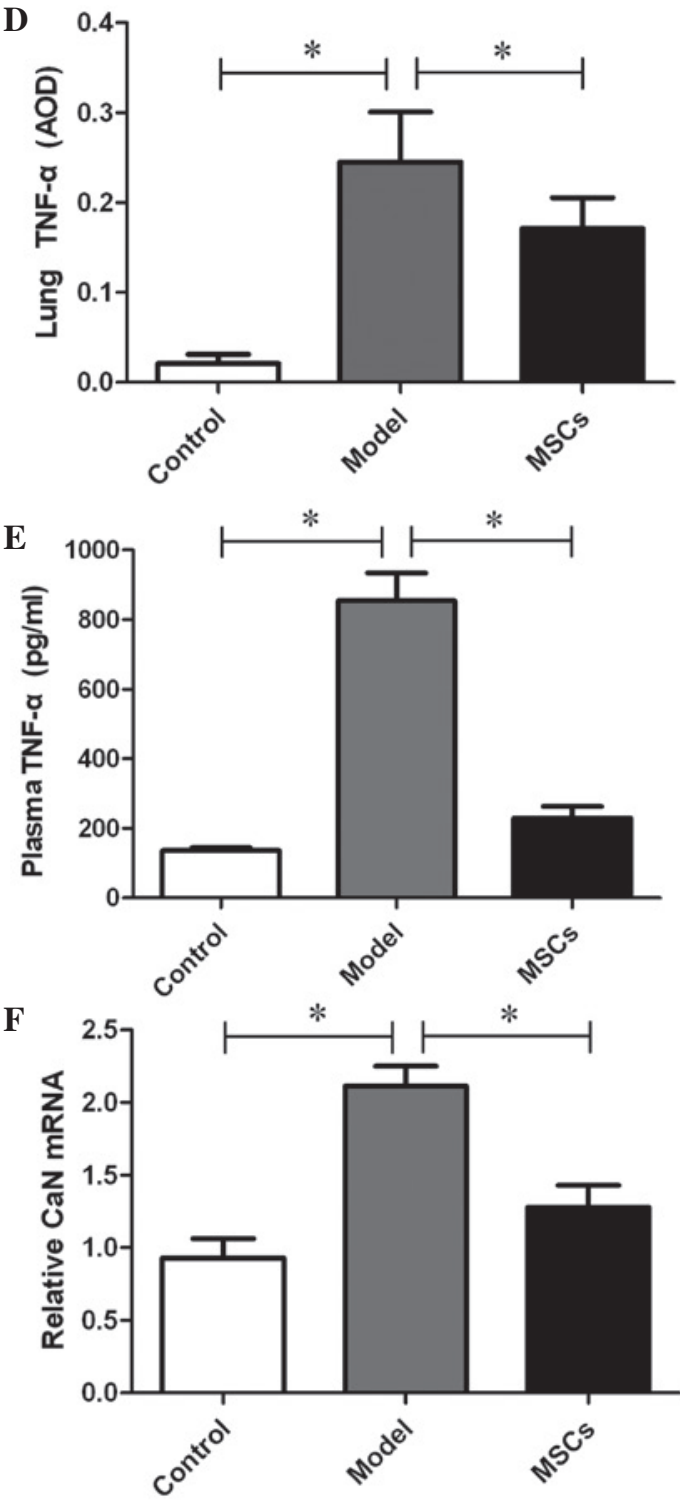

G

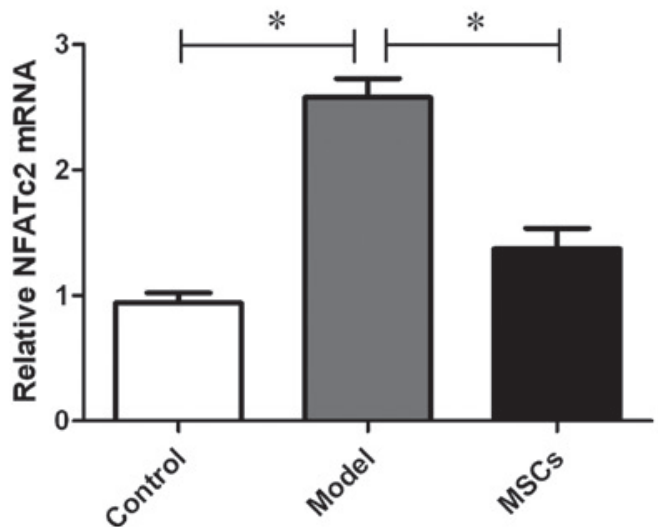

Figure 4. Effect of transplantation with mesenchymal stem cells (MSCs) on tumor necrosis factor (TNF)- $\alpha$, calcineurin (CaN) and nuclear factor of activated T-cells (NFATc2) expression levels. Immunohistochemical staining of lung tissue for TNF- $\alpha$ in (A) the control group, (B) the model group and (C) the MSC transplantation group. The expression levels of TNF- $\alpha$ in (D) the lung tissue and (E) the plasma of rats. (F) CaN and (G) NFATc2 expression in the pulmonary arteries of rats. Data are presented as the mean \pm standard deviation. $n=8$. ${ }^{*} \mathrm{P}<0.01$. AOD, average optical density.

study has demonstrated, it is possible that TNF- $\alpha$, which is produced by inflammatory cells that have infiltrated the lung tissue, may accelerate calcium mobilization and influx via specific channels in the membranes of PASMCs (10). Furthermore, increased levels of calcium in the cytoplasm may activate the proliferation of SMCs via the CaN/NFAT pathway (9). Therefore, inhibiting the production of TNF- $\alpha$ may be a potential treatment for $\mathrm{PH}$ as it may interrupt the activation of the CaN/NFAT pathway and thus prevent PASMCs hyperproliferation. 
As a subset of stromal stem cells, MSCs are widely distributed around the body and share the same biological characteristics, including the potential of multi-lineage differentiation and self-renewal. Previous studies have reported that MSCs have been harvested from the bone marrow (22), umbilical cord blood (23), the umbilical cord (17), the placenta (24), and adipose tissue (25) thus far. Umbilical cord-derived MSCs are particularly superior in terms of non-invasiveness, reduced donor harm/pain, and greater expansion capability $(17,26)$, which warrant the promising application of them in the future. However, in spite of the low immunogenicity and low potential for graft rejection of MSCs, as the traditional culture system is supplemented with FBS, the potential for zoonotic infection still exists with clinical application. Therefore, in the present study, the traditional culture medium for MSCs was exchanged for serum-free medium on the third passage, in order to decrease the effects of serum as much as possible. The results of the present study indicated that, under serum-free culture conditions, MSCs maintain their phenotype and similar growth characteristics. Thus, as MSCs retain the potential for multi-lineage differentiation, this could ensure the reliability of subsequent studies.

Successful localization to the injured lung tissue is the crucial first step in the treatment of $\mathrm{PH}$ with MSCs. Therefore, in the present study, CM-Dil was used to observe the distribution of MSCs in the lung tissue; the results of which demonstrated that, 2 days after the transplantation of the MSCs, an abundance of CM-Dil positive cells were localized in the lungs. This may suggest that MSCs have a protective effect in situ. The hypothesis that MSCs may differentiate into various lung tissue cell types after transplantation into a host with pulmonary diseases has been questioned; this is mainly due to a lack of a niche for MSCs during injury, which may be an obstacle to their engraftment or differentiation (27). A more acceptable hypothesis is that MSCs may have important cytoprotective effects, predominantly via various paracrine mechanisms (27). The authors of the present study have previously demonstrated that the ability of $\mathrm{CD}^{+} \mathrm{T}$ cells to produce $\mathrm{TNF}-\alpha$ was decreased when co-cultured with MSCs, and the immunosuppressive activity of MSCs is highly associated with their ability to secrete immunoregulatory cytokines and PGE2 (12). The present study has also demonstrated that in vivo exogenous transplantation of MSCs decreased the levels of TNF- $\alpha$ not only in the lung tissue but also in plasma by its immunosuppressive activity. Furthermore, the expression levels of CaN and NFATc2 in pulmonary arteries were downregulated; hence, the medial hypertrophy of the pulmonary muscular arterioles was improved, in addition to an alleviation of pulmonary artery pressure.

In conclusion, the present study demonstrates for the first time, to the best of our knowledge, that human umbilical cord-derived MSCs cultured in serum-free medium may suppress the hyperproliferation of PASMCs associated with PH. Furthermore, the regulating effect was accomplished by the immunosuppressive activity of MSCs, particularly via suppression of TNF- $\alpha$, and the subsequent inhibition of the CaN/NFAT pathway in PASMCs. However, the prospective efficacy and potential side-effects of these regulatory effects require confirmation in future studies.

\section{Acknowledgements}

The present study was supported by the National Natural Science Foundation of China (grant no. 30560159).

\section{References}

1. Frid MG, Brunetti JA, Burke DL, Carpenter TC, Davie NJ, Reeves JT, Roedersheimer MT, van Rooijen N and Stenmark KR: Hypoxia-induced pulmonary vascular remodeling requires recruitment of circulating mesenchymal precursors of a monocyte/macrophage lineage. Am J Pathol 168: 659-669, 2006.

2. Hall S, Brogan P, Haworth SG and Klein N: Contribution of inflammation to the pathology of idiopathic pulmonary arterial hypertension in children. Thorax 64: 778-783, 2009.

3. Pinto RF, Higuchi ML and Aiello VD: Decreased numbers of T-lymphocytes and predominance of recently recruited macrophages in the walls of peripheral pulmonary arteries from 26 patients with pulmonary hypertension secondary to congenital cardiac shunts. Cardiovasc Pathol 13: 268-275, 2004.

4. Heath D and Edwards JE: The pathology of hypertensive pulmonary vascular disease; a description of six grades of structural changes in the pulmonary arteries with special reference to congenital cardiac septal defects. Circulation 18: 533-547, 1958

5. Perros F, Dorfmüller P,Montani D, Hammad H, Waelput W, Girerd B, Raymond N, Mercier O, Mussot S, Cohen-Kaminsky S, et al: Pulmonary lymphoid neogenesis in idiopathic pulmonary arterial hypertension. Am J Respir Crit Care Med 185: 311-321, 2012.

6. Soon E, Holmes AM, Treacy CM, Doughty NJ, Southgate L, Machado RD, Trembath RC, Jennings S, Barker L, Nicklin P, et al: Elevated levels of inflammatory cytokines predict survival in idiopathic and familial pulmonary arterial hypertension. Circulation 122: 920-927, 2010.

7. de Frutos S, Spangler R, Alò D and Bosc LV: NFATc3 mediates chronic hypoxia-induced pulmonary arterial remodeling with alpha-actin up-regulation. J Biol Chem 282: 15081-15089, 2007.

8. Bonnet S, Rochefort G, Sutendra G, Archer SL, Haromy A, Webster L, Hashimoto K, Bonnet SN and Michelakis ED: The nuclear factor of activated $\mathrm{T}$ cells in pulmonary arterial hypertension can be therapeutically targeted. Proc Natl Acad Sci USA 104: 11418-11423, 2007.

9. Said SI, Hamidi SA and Gonzalez Bosc L: Asthma and pulmonary arterial hypertension: Do they share a key mechanism of pathogenesis? Eur Respir J 35: 730-734, 2010.

10. Rowlands DJ, Islam MN, Das SR, Huertas A, Quadri SK, Horiuchi K, Inamdar N, Emin MT, Lindert J, Ten VS, Bhattacharya $\mathrm{S}$ and Bhattacharya $\mathrm{J}$ : Activation of TNFR1 ectodomain shedding by mitochondrial $\mathrm{Ca}^{2+}$ determines the severity of inflammation in mouse lung microvessels. J Clin Invest 121: 1986-1999, 2011.

11. Wu M, Han ZB, Liu JF, Wang YW, Zhang JZ, Li CT, Xin PL, Han ZC and Zhu XP: Serum-free media and the immunoregulatory properties of mesenchymal stem cells in vivo and in vitro. Cell Physiol Biochem 33: 569-580, 2014.

12. Yang ZX, Han ZB, Ji YR, Wang YW, Liang L, Chi Y, Yang SG, Li LN, Luo WF, Li JP, et al: CD106 identifies a subpopulation of mesenchymal stem cells with unique immunomodulatory properties. PLoS One 8: e59354, 2013.

13. Le Blanc K, Rasmusson I, Sundberg B, Götherström C, Hassan M, Unzel M and Ringdén O: Treatment of severe acute graft-versus-host disease with third party haploidentical mesenchymal stem cells. Lancet 363: 1439-1441, 2004.

14. Zappia E, Casazza S, Pedemonte E, Benvenuto F, Bonanni I, Gerdoni E, Giunti D, Ceravolo A, Cazzanti F, Frassoni F, Mancardi G and Uccelli A: Mesenchymal stem cells ameliorate experimental autoimmune encephalomyelitis inducing T-cell anergy. Blood 106: 1755-1761, 2005.

15. BaberSR, Deng W, MasterRG,BunnellBA, Taylor BK, Murthy SN, Hyman AL and Kadowitz PJ: Intratracheal mesenchymal stem cell administration attenuates monocrotaline-induced pulmonary hypertension and endothelial dysfunction. Am J Physiol Heart Circ Physiol 292: H1120-H1128, 2007.

16. Luan Y, Zhang X, Kong F, Cheng GH, Qi TG and Zhang ZH: Mesenchymal stem cell prevention of vascular remodeling in high flow-induced pulmonary hypertension through a paracrine mechanism. Int Immunopharmacol 14: 432-437, 2012. 
17. Lu LL, Liu YJ, Yang SG, Zhao QJ, Wang X, Gong W, Han ZB, Xu ZS, Lu YX, Liu D, Chen ZZ and Han ZC: Isolation and characterization of human umbilical cord mesenchymal stem cells with hematopoiesis-supportive function and other potentials. Haematologica 91: 1017-1026, 2006.

18. Matsuda Y, Hoshikawa Y, Ameshima S, Suzuki S, Okada Y, Tabata T, Sugawara T, Matsumura Y and Kondo T: Effects of peroxisome proliferator-activated receptor gamma ligands on monocrotaline-induced pulmonary hypertension in rats. Nihon Kokyuki Gakkai Zasshi 43: 283-288, 2005 (In Japanese).

19. Maruyama H, Watanabe S, Kimura T, Liang J, Nagasawa T, Onodera M, Aonuma K and Yamaguchi I: Granulocyte colony-stimulating factor prevents progression of monocrotaline-induced pulmonary arterial hypertension in rats. Circ J 71: 138-143, 2007.

20. Zangiabadi A, De Pasquale CG and Sajkov D: Pulmonary hypertension and right heart dysfunction in chronic lung disease. Biomed Res Int 2014: 739674, 2014.

21. Patel R, Aronow WS, Patel L, Gandhi K, Desai H, Kaul D and Sahgal SP: Treatment of pulmonary hypertension. Med Sci Monit 18: RA31-RA39, 2012.

22. Pittenger MF, Mackay AM, Beck SC, Jaiswal RK, Douglas R, Mosca JD, Moorman MA, Simonetti DW, Craig S and Marshak DR: Multilineage potential of adult human mesenchymal stem cells. Science 284: 143-147, 1999.
23. Lee OK, Kuo TK, Chen WM, Lee KD, Hsieh SL and Chen TH: Isolation of multipotent mesenchymal stem cells from umbilical cord blood. Blood 103: 1669-1675, 2004.

24. Fukuchi Y, Nakajima H, Sugiyama D, Hirose I, Kitamura T and Tsuji K: Human placenta-derived cells have mesenchymal stem/progenitor cell potential. Stem Cells 22: 649-658, 2004.

25. Zuk PA, Zhu M, Mizuno H, Huang J, Futrell JW, Katz AJ, Benhaim P, Lorenz HP and Hedrick MH: Multilineage cells from human adipose tissue: Implications for cell-based therapies. Tissue Eng 7: 211-228, 2001.

26. Baksh D, Yao R and Tuan RS: Comparison of proliferative and multilineage differentiation potential of human mesenchymal stem cells derived from umbilical cord and bone marrow. Stem Cells 25: 1384-1392, 2007.

27. Jun D, Garat C, West J, Thorn N, Chow K, Cleaver T, Sullivan T, Torchia EC, Childs C, Shade T, et al: The pathology of bleomycin-induced fibrosis is associated with loss of resident lung mesenchymal stem cells that regulate effector T-cell proliferation. Stem Cells 29: 725-735, 2011. 